\title{
Negative modulation of human osteoclastogenesis by antiepileptic drugs
}

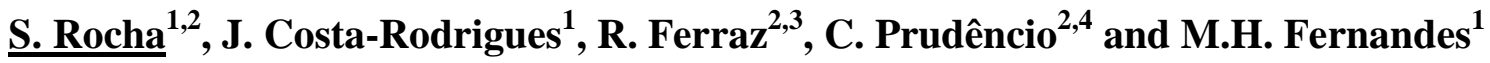 \\ ${ }^{1}$ Laboratory for Bone Metabolism and Regeneration, Faculty of Dental Medicine, University of \\ Porto, Portugal. \\ ${ }^{2}$ CISA/CQB Escola Superior de Tecnologia da Saúde do Porto Instituto Politécnico do Porto, \\ Portugal. \\ ${ }^{3}$ REQUIMTE-CQFB, Faculdade de Ciências e Tecnologia da Universidade Nova de Lisboa, \\ Portugal. \\ ${ }^{4}$ Centro de Farmacologia e Biopatologia Química (U38-FCT), Faculty of Medicine, University \\ of Porto, Portugal.
}

Bone is constantly being molded and shaped by the action of osteoclasts and osteoblasts. A proper equilibrium between both cell types metabolic activities is required to ensure an adequate skeletal tissue structure, and it involves resorption of old bone and formation of new bone tissue. It is reported that treatment with antiepileptic drugs (AEDs) can elicit alterations in skeletal structure, in particular in bone mineral density. Nevertheless, the knowledge regarding the effects of AEDs on bone cells are still scarce, particularly on osteoclastic behaviour. In this context, the aim of this study was to investigate the effects of five different AEDs on human osteoclastic cells.

Osteoclastic cell cultures were established from precursor cells isolated from human peripheral blood, and were maintained in the absence (control) or in the presence of $10^{-}$ ${ }^{8}-10^{-4} \mathrm{M}$ of different AEDs (valproate, carbamazepine, gabapentin, lamotrigine and topiramate). Cell cultures were characterized throughout a 21-day period for tartrateresistant acid phosphatase (TRAP) activity, number of TRAP+ multinucleated cells, presence of cells with actin rings and expressing vitronectin and calcitonin receptors, and apoptosis rate. Also, the involvement of several signaling pathways on the cellular response was addressed.

All the tested drugs were able to affect osteoclastic cell development, although with different profiles on their osteoclastogenic modulation properties. Globally, the tendency was to inhibit the process. Furthermore, the signaling pathways involved in the process also seemed to be differentially affected by the AEDs, suggesting that the different drugs may affect osteoclastogenesis through different mechanisms.

In conclusion, the present study showed that the different AEDs had the ability to negatively modulate the osteoclastogenesis process, shedding new light towards a better understanding of how these drugs can affect bone tissue. 